\title{
The Application of Virtual Reality Technology in Smart Hom
}

\author{
Phonesouda Souphamith and Lasheng $\mathrm{Yu}^{2, *}$ \\ ${ }^{1}$ School of Computer Science and Engineering, Central South University Changsha, China \\ ${ }^{2}$ School of Computer Science and Engineering, Central South University Changsha, China \\ *Corresponding author
}

\begin{abstract}
In recent years, smart home is a versatile system that includes visual intercom, home security, remote monitoring of home appliances, remote video surveillance, telemedicine diagnostics and care systems, online education systems and home movie start systems. The basic goal of smart home is to connect various information-related communication devices, household appliances and home security devices in the home to an intelligent system for centralized or remote monitoring, control and family affairs management, and to maintain these home facilities and Harmony and coordination of the residential environment. At the same time, virtual reality technology and three-dimensional technology are becoming more and more mature, and begin to be applied in various industries. Three-dimensional virtual reality has broken the traditional understanding of computer humancomputer interaction in the past. The way people receive computer information has changed from data two-dimensional images to three-dimensional virtual models. User interaction mode of traditional smart home ranges from two-dimensional control interface to three-dimensional virtual. Control in the scene has become the future development trend of smart home. This paper develops a virtual smart home system, which successfully combines three-dimensional technology with smart home system. This paper designs the system architecture and functional modules of the virtual smart home system, designs and analyses the workflow of each module, and combines the key technologies of the virtual smart home system, such as modeling technology, roaming technology, node control method and so on.
\end{abstract}

Keywords-3D virtual reality model of computer application smart home

\section{INTRODUCTION}

VITURAL INTERFACE is a comprehensive integration technology, involving computer graphics, human-computer interaction technology, sensor technology, artificial intelligence and other fields. It generates realistic three-dimensional visual, auditory, olfactory and other senses by computer, so that people as participants can experience and interact with the virtual world naturally through appropriate devices. When the user moves the position, the computer can immediately perform complex calculations, and send back accurate 3D world images to create a sense of presence. This technology integrates the latest developments of computer graphics (CG), computer simulation, artificial intelligence, sensor technology, display technology and network parallel processing technology. It is a high-tech simulation system generated by computer technology. A kind of. Generally speaking, virtual reality is a new way for people to visualize and interact with complex data through computers. Compared with traditional humancomputer interface and popular window operation, virtual reality has made a qualitative leap in technical thinking. The "reality" in virtual reality refers to anything or environment that exists in the world in a physical or functional sense. It can be realizable in practice, or it can be difficult or impossible to achieve at all. And "virtual" refers to the meaning generated by computer. Therefore, virtual reality refers to a special environment generated by computer. People can "project" themselves into this environment by using various special devices, and operate and control the environment to achieve a special purpose, that is, people are the dominant part of this environment. Specifically, virtual reality interaction technology is to use modern high-tech technology with computer technology as the core to generate a realistic visual, auditory and tactile integration of specific range of virtual environment. Users interact and interact with the objects in the virtual environment in a natural way with the necessary equipment, so as to produce feelings and experiences equipment to the real environment in person. So how did this virtual real world come into being? Firstly, we need to generate virtual entities, which are mainly aimed at the user's physiological sense. Secondly, users interact with the environment through the natural skills of human physiology, finally, we use sensors to complete the interaction between human and virtual environment.

\section{The Proposed ViRTUAL REALITy System}

\section{A. System Design Rationale}

The virtual home system can be divided into three main components. They are virtual reality client, virtual reality sever and IOS mobile phone control. In this system, the virtual reality client is in the three-dimensional display part, and the user can traverse the virtual scene through free-view roaming or fixedview preview. The virtual reality client is implemented through three-dimensional modeling, lighting environment dyeing, and three-dimensional virtual interaction. The virtual reality client provides controllable nodes for many appliances, such as contempt, air conditioning, water heaters, lighting, etc. while the user feels the virtual home environment, it can also be controlled through the provided control interface. The control command is uploaded to the server. The server parses the command. The virtual scene is then sent through a request. Like the sever requests data. Through the returned number. After parsing, modify the control node corresponding to the 
virtual scenes. This process realizes th e communication between the virtual scene and the server. The databases of the virtual reality server side records the stat data of each controllable node in the three-dimensional scene. The mobile phone control point utilizes the interface provided by the server. It directly sends control commands to modify the fields of the database. The 3D virtual reality client modifies the scene by requesting database fields. Therefore, this communication method can make the mobile phone control terminal implemented control of virtual scene such as TV switches, air conditioning temperatures, etc.

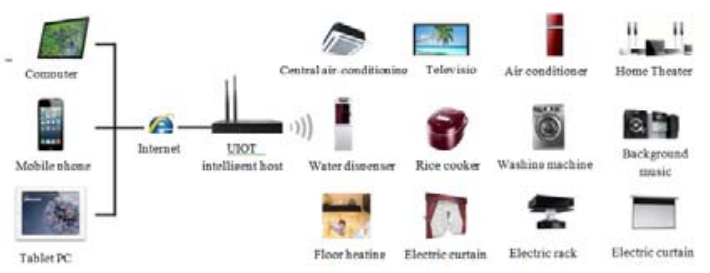

FIGURE I. VIRTUAL HOME SYSTEM

\section{B. Virtual Reality Server}

The virtual reality server uses the Apache server and the MySQL database. Apache server the most used web server software in the world. Its advantages are cross-platform and security. And it can be fast, reliable, and con be compiled into a server with a simple API extension. Many scripting language interpreters are co mpiled into the server . MySQL [11] is a relational databases management system. It is one of the most popular database applications. The most popular W MAP network development architecture is the MySQL database with Apache and PHP server scripting language.

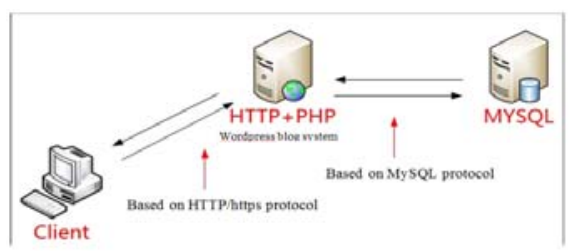

FIGURE II. VIRTUAL REALITY SERVE

\section{IOS Moblile Phone Console}

IOS $[12,15]$ is a mobile operating system developed by apple. The IOS application can be found on Apple's iPhone, iPod, iPad. Due to increasing popularity of Apple devices, the system uses the IOS mobile phone control terminal as example. The interface provided by the ser ver is used for control terminal development. Of course, the development of the Android console is also allowed. Because the system provides a development interface. Tool name 3dsMax 2012, Unity3D 4.2.1, Photoshop CS6, Win64 bit operating system use to establish 3D model, 2D development engine, image processing, system development, operation platform whole system consists of three layers: presentation layer, logic layer and data layer. The data layer is the lowest part. This section stores data for the model's data and spatial information. Among them, the model data is designed with reference to the size and size of the actual home. It includes various devices such as TV, air conditioner and other el ectrical appliances. By actu ally measuring and referencing the CAD drawings of the building, we get the real data and draw the model based on the data. This allows the size of the model to match the actual situation. I ( $\mathrm{r} 1$ space information data includes terrain, light intensity, temperature and humidity, weather, etc. these can obtained through the sensor to obtain real data. Then through the engine simulation. This can make the virtual scene environment and real IOS; Use) "Experience". The logic layer includes the level of the model and the logic of the Unity object. The logic is t hat the development becomes convenient and fast. The parent -child relationship of the model level makes it easier to control the object when designing the interaction. For example, when moving a computer, it will not move the mouse alone. It will move to the desired position as a whole without destroying the internal structure of the model. Unity's object logic is more complicated. Each object of Unity has its own components. These components including material information, position coordinates, angle size, etc. at the same time, Unity objects can be packaged into resources to make them universal.

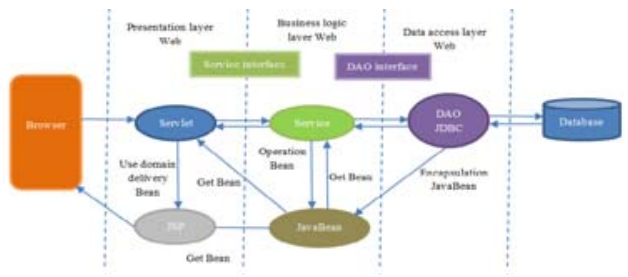

FIGURE III. VIRTUAL REALITY SERVER

\section{Three-dimenional Modeling}

In the act ual modeling process, the relationships between these layers are interspersed. For example, there ar e multiple equipment layers and vegetation layers under each building. 3D modeling is done using 3dsMax. However, in actual operation, a single building or de vice model is often built by 3 dsMax. Then the model files are exported. These model file resources are respectively poured into Unity3D. In the Unity3D engine, assembly and de bugging are per formend. So s cene stratification is very important. The $3 \mathrm{D}$ interaction uses the Unity3D engine. The three-dimensional interaction of the virtual scene can be divided into four parts: scene roming, node control, information query and virt ual monitoring. Scene roaming can be divided into two types of roaming methods: first person roaming and fixed angle roaming. The first person roaming is from a mountain perspective. The user can use the mouse and keyboard to control the movement and visibility of the character. The same angle of view roaming provides a fixed perspective. It is convenient for users to perform node control and information query.

\section{EXPERIMENT AND RESULTS}

The research content of this paper is to study the application of 3D technology in s mart home systems. Using modeling technology, 3D interactive technology, roaming technology and other technical means. Using 3dsMax. Unity3D and other development tools. Development a virtual smart home system. 


\section{A. System Implemention}

The virtual smart home system provides a new solution for the development of smart homes. The sy stem starts from the functional requirements of the current smart home. It simulates the common functions of the smart home system and the functions to be developed in the future. The realization of the control function is repres ented by the change of the threedimensional virtual scene. The front-end 3D virtual scene of the virtual smart home system intuitively displays the virtual smart home model. It provides first-person roaming. It allows users to be physically present. At the same time, it provides a controllable node control menu for use rs to control the controllable devices. The virtual smart home system background server provides communication connection service. At the same time, the database records the user operation and the status data of the virtual smart home appliance. The server provides a control interface for external development and use. The mobile intelligent control system of the virtual smart home system directly controls the controllable node model in the virtual scene through the IOS device, and achieves the purpose of remote control.

- Implementation plan: $\mathrm{t}$ he core par $\mathrm{t}$ of the virtual installation system is the control of the 3D scene camera and the movement of the object on the three axes of xyz. The corresponding table design corresponding to the camera is show in table 1

\section{TABLE I. CAMERA CONTROL}

\begin{tabular}{|l|l|}
\hline Input & response \\
\hline Alt+lefl|mouse button & The camera rotates around the current target point \\
\hline Alt+mouse wheel & Zoom in or Zoom out of the camera \\
\hline Alt + mouse middle button & Moving camera position \\
\hline
\end{tabular}

The purpose of adding the Alt key is to distinguish between the selected smart home components and the control camera, and the table design corresponding to the input and response of the smart home components is show in table 2:

TABLE II. OBJECT CONTROL

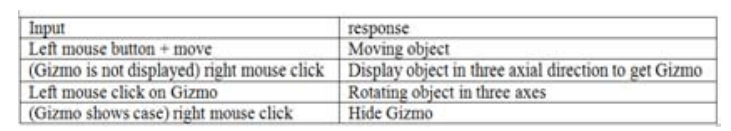

In terms of user interface, I use N GUI's camera to render all UI interface elements, because NGUI's control event response requires NGUI's camera support. At the same time, in order to render the 3D scene, an additional 3D camera needs to be set up

- Implementation of virtual installation interface: the virtual installation scenario is the core part of the system. The experimenter will experience the interaction with virtual object in this s cene, and complete the experiment of assembling the desktop smart home. Build a v irtual a virtual installation scenario. According to the model that was not imported into 3dsMax from the outside of this article to Unity, Unity will automatically generate the shared. After specifying the material, you need to add lights to the scene. Because it is an indoor scene, it is mainly based on point lighting. The scene renderings. Are shown

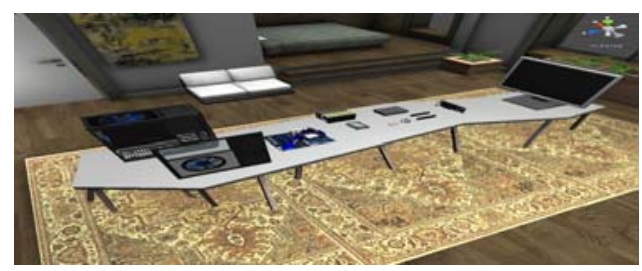

FIGURE IV. SMART HOME SCENE

Since it is necessary to interact with objects in the virtual world, collision detection between the mouse and the object is required, and when the mouse touches and objects in the virt ual world, the vir tual object responds. There are $t$ hree ways to do co llision detection in Unity. The first is a collider, the second is a trigger, and the last is radiography. Colliders are a group of components that contain many types, such as box collider, capsule collider, etc. these colliders have different applications but have the same effect. A trigger means that an ev ent is triggered when a collision occurs. To add a trigger, just check the Istrigger option in the monitor window of the collider. In Unity3d, collision detection of colliders and triggers is handled primarily through the interface show in figure 5

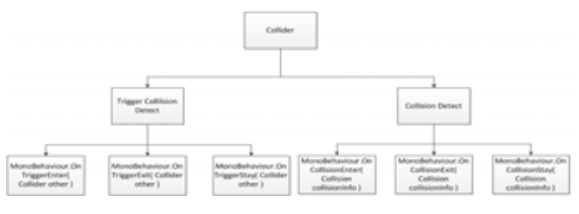

\section{FIGURE V. COLLIDER INTERFACE IN UNITY}

Specifically, the colliders can be divided into three categories. A static collider refers to an object with a collider attached without a rigid body attached. Such object will remain stationary or move very slightly. A Rigidbody collider refers to an object with rigid body attached. The kinematic Rigidbody collider refers to a collider with the IsKinematic attribute selected in the rigid body assembly. It will not be affected by physical forces. The message that different colliders send to the collision class object when collision detection occurs is show in table 3:

TABLE III. SENDING INFORMATION TABLE DURING COLLIDER DETECTION

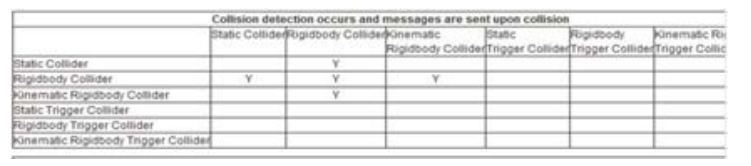

The message that different colliders send to the collision class object when the trigger fires. 
TABLE IV. SENDING A MESSAGE TABLE WHEN THE TRIGGER IS TRIGGERED

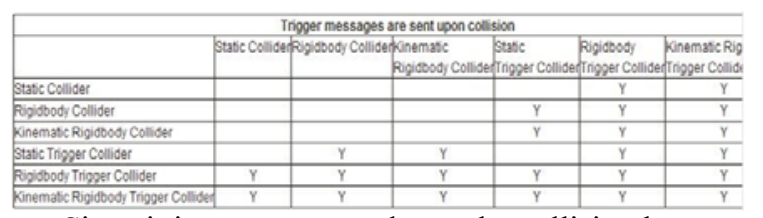

Since it is necessary to detect the collision between the screen point where the player mouse is located and the scene object, and the mouse cannot add the collider, the ray method is used here for collision. It principle is to draw a certain length of ray's perpendicular to the screen form the current mouse point as a starting point, and mark the object through which the ray passes. The ray collision detection code used in this experiment each smart home component in the scene has three scripts ObjectMoveControl, Hit, and DistanceDetect to control movement, rotation, and distance detection. Their relationship is show in figure 6:

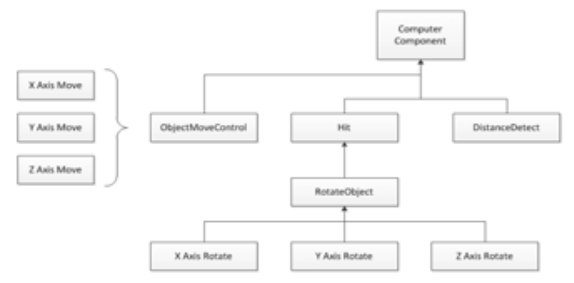

FIGURE VI. CONTROL OF SMART HOME COMPONENTS

\section{B. System Evaluation}

The virtual smart home system test from the aspects of roaming function, 3D client control function, mobile control terminal control function and virtual monitoring. In the roaming function, the $\mathrm{r}$ ole control function and collision detection function of the system are tested, the threedimensional client control function and the control function of the mobile phone control terminal have certain similarities. The functions of the control nodes such as TV, air conditioner, water heater, and desk lamp are respectively controlled. Test. Finally, the application test was performed on the virtual monitoring function of the $3 \mathrm{D}$ client. The test show that the virtual smart home system can work normally. It also verifies the successful application of 3D technology in smart home systems. This system is virtual assembly smart home system based on Unity3D engine and developed by virtual reality technology. With Unity3d's powerful graphics rendering pipeline support, you ca $\mathrm{n}$ get ver y realistic environment simulation and virtual operation simulation.

\section{The Real-user Experiment}

The virtual smart home system simulates the real home environment through virtual reality technology. It displays the living environment of people on the user's screen. It makes people immersive. It also provides some controllable nodes. It allows user to freely control virtual home appliances.

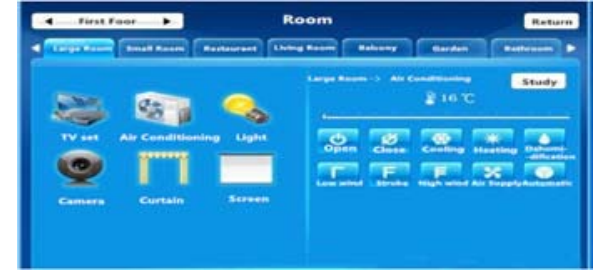

FIGURE VII. VIRTUAL HOME CONTROL

The mobile phone control end node provides a contro 1 operation of a part of the $t$ hree-dimensional virtual scene controllable node. Includes table lamp switch control, humidifier switch control, curtain switch control, TV control, air conditioning control and virtual air detection. The server interface used by the mobile phone control terminal is consistent with the three-dimensional client control interface. This article takes the mobile TV terminal to control the virtual TV as example to test the control function of the mobile phone control terminal.

\section{CONCLUSION AND FUTURE WORKS}

We have learned a lot of relevant knowledge in the process of researching this topic and realizing the smart home system. We also found many deficiencies, such as the lack of friendly system interface and the improvement of the overall model wring. The function of the smart home system to be continuously improved. In add ition, the stability and antiinterference of the system still need to be improved. The embedded development technology based on arm is applied to smart home, and the embedded remote intelligent home monitoring system based on arm is designed.

The future of smart home is geared toward the direction of intelligence and humanity. With the $\mathrm{i}$ ntegration and transformation of 3D technology, all kinds of industries and smart homes are also the future development trend. The virtual smart home system researched in this paper is to integrate $3 \mathrm{D}$ technology into the virtual smart home system. There are still some areas for improvement in practical applications. If it is not combined with real hardware systems, the more significance of this paper is new at tempt is not very practical. The smart home system based on real hardware based on 3D technology need further research and development.

\section{ACKNOWLEDGMENT}

The authors would like to say thank you to the anonymous referees for their helpful comments and suggestion.

\section{REFERENCES}

[1] Tu Y, Lei Y, Li H, et al. An augmented smart home system based on the internet of people concept[C]// Telecommunication Networks \& Applications Conference. IEEE, 2017.

[2] Yang K M, Cho S B. STRIPS Planning w ith Modular Behavior Selection Networks for Smart Home Agents[C]// IEEE International Conference on Ubiquitous Intelligence \& Computing \& I EEE International Conference on Autonomic \& Trusted Computing. IEEE Computer society, 2013.

[3] Nguyen T, K im J G , Choi D. ISS: The Interactive smart-home Simulator[C]// International Conference on Advanced Communication Technology. IEEE Press, 2009. 
[4] Du K K, Wang Z L, Hong M. Human machine interactive system on smart home of IoT [J]. The Journal of China Universities of Posts and Telecommunication, 2013, 20(Supple 1):96-99.

[5] Van Nguyen T, Nguyen H, Choi D. Development of a Context Aware Virtual Smart home Simulator $[\mathrm{J}]$. Computer Science, 2010 , abs/1007.1274.

[6] Ploh J. Comparison of Unity3D and Construct 2 tools for development of games on mobile platforms [J]. 2014

[7] Brooker D. Essential CG Lighting Techniques with 3ds Max [J]. Taylor \& Francis Ltd, 2013: iv.

[8] Steed P, Steed P. Modeling a character in $3 \mathrm{ds} \mathrm{m}$ ax, second edition [J]. Folia Parasitologica, 2014, 62(64).

[9] Wang Zhenggang Reflections on the Application of 3dsMax Teaching [J]. Decoration, 2005(7):18-18.

[10] Lei Z, Taghaddos H, Han S H, et al. From AutoCAD to 3ds Max: An automated approach for animating heavy lifting studies [J]. Canadian Journal of Civil Engineering, 2015, 42(3):190-198.

[11] Bellia L, Pedace A, Fragliasso F. The impact of the software's choice on dynamic daylight simulations' results: A c omparison between Daysim and 3ds Max Design? [J]. Solar Energy, 2015, 122:249-263. Ma Siqun, Li Shasha, Yang Wei, et al. Design of VIRTUAL INTERFACE System for High Seepd Train Based on 3Ds Max and VITURAL INTERFACEP [J]. Computer Simulation, 2013, 30(1).

[12] Peng Guohua. Research and Application of 3D Animation Character Modeling Technology Based on 3ds Max [J]. Journal of Shaanxi University of Science and Technology (Natural Science Edition), 2010, 28(2).

[13] Li Zhengzhou. 3D Magic: Cult 3D \& 3DSMAX [M]. Publishing House of Electronics Industry, 2002.

[14] Fan Jun, Wan Lin Tang Yulin. Calculation of Target Intensity in Underwater Based on 3DS MAX Modeling [J]. Journal of Acoustics, 2000, 19(4): 203-204.

[15] Liu J, Jiang H. Development of a virtual winder for computer-aided education using Virtools [J]. Computer Applications in Engineering Education, 2014, 22(1):120-130.

[16] Chaudhuri S, Koltun V. Data-driven suggestions for creativity support in 3D modeling [J]. ACM Transactions on Graphics, 2010, 29(6):1.

[17] Li Zhenghao. 3D Magic: Cult 3D \& 3DSMAX [M]. Publishing House of Electronics Industry, 2002.

[18] Reinhart C, Breton P F. Experimental Validation of Autodesk (R) 3ds Max (R) Design 2009 and Daysim 3.0[J]. LEUKOS. The Journal of the Illuminating Engineering Society of North America, 6(1).

[19] Xu K, Chen K, Fu H, et al. Sketch2scene: Sketch-based co-retrieval and co-placement of 3d models [J]. Acm Transactions on Graphics, 2013, 32(4):1-15.

[20] Xu G, Piao D, Musgrove C H, et al. Trans-rectal ultrasound-coupled near-infrared optical tomography of the prostate, part I: simulation. [J]. Optics Express, 2008, 16(22):17484-17504.

[21] Secord A, Lu J, Finkelstein A, et al. Perceptual models of viewpoint preference [J]. ACM Transactions on Graphics, 2011, 30(5):1-12.

[22] Pan J, Xing L X, Li B S, et al. Application of three-dimensional visualization in simulation of geographical landscape evolvement [J]. Chinese Geographical Science, 2005, 15(2):168-172.

[23] Passos C, Márcio H. da Silva , Mol A C A, et al. Design o f a collaborative virtual environ ment for training securi ty agents in big events [J]. Cognition Technology \& Work, 2017.

[24] Avilagh A J, Rezaie A H. Accuracy enhancement of three-dimensional reconstruction using phase-shifting shadow moiré [J]. Journal of Biological Chemistry, 2013, 279(30):31259-31267.

[25] Nebel J C, Sibiryakov A, Ju X. V-Man generation for 3-D real time animation [J]. Plant Cell, 2003, 17(5):1625-36.

[26] IVANCEVIC, VLADIMIR. LIE-LAGRANGIAN MODEL FOR REALISTIC HUMAN BIODYNAMICS [J]. International Journal of Humanoid Robotics, 2006, 03(02):205-218 